\title{
A 3D printed drug delivery implant formed from a dynamic supramolecular polyurethane formulation
}

Article

Accepted Version

Salimi, S., Wu, Y., Evangelista Barreiros, M. I., Natfji, A. A., Khaled, S., Wildman, R., Hart, L. R., Greco, F., Clark, E. A., Roberts, C. J. and Hayes, W. (2020) A 3D printed drug delivery implant formed from a dynamic supramolecular polyurethane formulation. Polymer Chemistry, 11 (20). pp. 3453-3464. ISSN 1759-9954 doi: https://doi.org/10.1039/D0PY00068J Available at https://centaur.reading.ac.uk/90468/

It is advisable to refer to the publisher's version if you intend to cite from the work. See Guidance on citing.

To link to this article DOI: http://dx.doi.org/10.1039/D0PY00068J

Publisher: Royal Society of Chemistry

All outputs in CentAUR are protected by Intellectual Property Rights law, including copyright law. Copyright and IPR is retained by the creators or other copyright holders. Terms and conditions for use of this material are defined in the End User Agreement. 


\section{CentAUR}

Central Archive at the University of Reading

Reading's research outputs online 


\title{
A 3D Printed Drug Delivery Implant formed from a Dynamic Supramolecular Polyurethane Formulation
}

\author{
S. Salimi, ${ }^{\text {a }}$ Y. Wu, ${ }^{\text {b, }}$ M. I. Evangelista Barreiros, ${ }^{\text {b }}$ A. A. Natfji, ${ }^{c}$ S. Khaled, ${ }^{\text {d }}$ R. Wildman, ${ }^{b}$ L.R. Hart, ${ }^{a}$ \\ F. Greco, ${ }^{c}$ E. A. Clark, ${ }^{\mathrm{d}}$ C. J. Roberts ${ }^{* \mathrm{~d}}$ and W. Hayes*a \\ a Department of Chemistry, University of Reading, Whiteknights, Reading, RG6 6AD, UK email: w.c.hayes@reading.ac.uk. \\ ${ }^{b}$ Faculty of Engineering, The University of Nottingham, University Park, Nottingham, NG7 2RD, UK \\ ${ }^{\mathrm{c}}$ School of Pharmacy, University of Reading, Whiteknights, Reading, RG6 6AD, UK \\ ${ }^{\mathrm{d}}$ School of Pharmacy, University of Nottingham, Nottingham NG7 2RD, UK
}

\begin{abstract}
Using a novel molecular design approach, we have prepared a thermo-responsive supramolecular polyurethane as a matrix material for use in drug eluting implants. The dynamic supramolecular polyurethane (SPU) is able to self-assemble through hydrogen bonding and $\pi-\pi$ stacking interactions, resulting in an addressable polymer network with a relatively low processing temperature. The mechanical properties of the SPU demonstrated the material was self-supporting, stiff, yet flexible thus making it suitable for hot-melt extrusion processing, inclusive of related 3D printing approaches. Cellbased toxicity assays revealed the SPU to be non-toxic and therefore a viable candidate as a biocompatible polymer for implant applications. To this end, the SPU was formulated with paracetamol $(16 \% \mathrm{w} / \mathrm{w})$ and $4 \mathrm{wt} \%$ or $8 \mathrm{wt} \%$ poly(ethylene glycol) (PEG) as an excipient and hot melt extruded at $100{ }^{\circ} \mathrm{C}$ to afford a 3D printed prototype implant to explore the extended drug release required for an implant and the potential manipulation of the release profile. Furthermore, rheological, infra-red spectroscopy, powder X-ray diffraction and scanning electron microscopy studies revealed the chemical and physical properties and compatibility of the formulation components. Successful release of paracetamol was achieved from in vitro dissolution studies and it was predicted that the drug would be released over a period of up to 8.5 months with hydrophilic PEG being able to influence the release rate. This extended release time is consistent with applications of this novel dynamic polymer as a drug eluting implant matrix.
\end{abstract}

\section{Introduction}

Investigating new functional excipient materials for local or systemic delivery of drugs is of ongoing interest. ${ }^{1}$ Such materials can lead to a range of benefits that can include improved efficiency of drug delivery, reduced toxicity and side effects and increased patient compliance. ${ }^{2}$ A key consideration when designing implantable drug delivery devices is the mechanism(s) and hence kinetics by which the desired drug is released. This can for example, be through physical degradation of a matrix, desorption 
from a matrix surface, diffusion of the drug from within a matrix, or cleavage of a chemical bond to liberate the free drug. ${ }^{3}$ The ideal release time can range from hours to months depending on the condition being treated, the active ingredient, and the route of delivery and it is hence desirable that release rate is relatively easily manipulated via any newly developed matrix material.

Materials developed for potential drug delivery applications range from porous materials, ${ }^{4}$ such as metal-organic frameworks, ${ }^{5,6}$ to polymers and gels $^{7-11}$ which release the drug upon exposure to a suitable stimulus (e.g. $\mathrm{pH}$ and temperature). ${ }^{12-14}$ Polymeric systems typically comprise of polyurethanes, ${ }^{15}$ poly(caprolactone), ${ }^{16}$ poly(dimethylsiloxane), ${ }^{17}$ poly(lactic acid) ${ }^{18}$ and more extensively polyethylene glycol (PEG) ${ }^{13,19-22}$ Additionally, blends of different classes of polymers have been used ${ }^{22-24}$ and are employed to modify or tune release rates and profiles through a combination of the individual components properties. In the majority of polymeric drug delivery implant systems, release occurs upon degradation (surface or bulk) of the polymer matrix, resulting in increased surface area and subsequent drug release. ${ }^{25}$ A prominent example of this is the slow hydrolysis of carbamate motifs within polyurethanes which makes them suitable for long-term drug release in implants. ${ }^{21}$ This rate of bond cleavage and hence drug release can be tuned by blending polymers with different degradation rates.

One new approach being investigated to produce different types of solid dosage forms is to deposit the formulation using additive manufacturing processes such as 3D printing of solutions via ink-jet technologies ${ }^{26}$ and solids by extrusion techniques. ${ }^{27}$ The later has shown promise in the 3D printing of well-known pharmaceutical materials, owing to similarities with traditional processing methods such as hot-melt extrusion. ${ }^{28}$ However, high processing temperature $\left(\geq 180{ }^{\circ} \mathrm{C}\right)$ involved in this method, constraint the components that can be used to formulate pharmaceuticals. ${ }^{29,30} \mathrm{We}$ offer a new approach to the formation of the solid implant from extrusion-based approaches at relatively mild temperatures. Herein, a dynamic supramolecular polyurethane (SPU) is reported which is able to self-assemble through hydrogen bonding and $\pi-\pi$ stacking interactions, ${ }^{31}$ giving rise to thermally reversable material properties. ${ }^{32-34}$ The thermally addressable nature of the SPU makes it an ideal candidate for hot-melt extrusion, inclusive of 3D printing approaches, as the melt viscosity is in a suitable range for printing and a stiff, yet flexible structure is rapidly produced upon cooling. ${ }^{35}$ The relatively low temperature required to dissociate the supramolecular interactions, and thus deposit the material, may also widen the range of actives available to be formulated as a result of the mild processing conditions preventing thermal degradation. This lower operating temperature could address one of the main obstacles regarding extrusion of pharmaceutical formulations. Additionally, the release profile of a drug (paracetamol) can be modulated and enhanced by co-formulating with PEG, ${ }^{36,37}$ which is a regulatory approved and commonly used as an excipient in pharmaceutical and personal care applications. As a widely used pain-killer, it may be advantageous to incorporate paracetamol into an implant (e.g. for knee and joint replacement applications). The extrusion 3D printing of SPU-PEG-paracetamol 
formulations to produce robust constructs which demonstrated suitable mechanical performance as well as biocompatibility is reported herein. Drug release from the printed formulations was evaluated via in vitro studies and the extrapolation of this data predicted that a release duration of 5 to 8.5 months would be achievable with the formulations; a timescale range that is consistent with many implant applications. There is clear potential to alter release rates further through modification of the formulation by varying the percentage of PEG or its molecular weight.

\section{Results and Discussion}

In order to create a prototype biomedical implant-style device which can be fabricated by hot-melt extrusion 3D printing, a thermally reversible polymer with a melting point below $140{ }^{\circ} \mathrm{C}$ (as dictated by the printer being used) was required. By harnessing the reversible nature of the supramolecular recognition motifs, the disassembly of the polymer network could be thermally triggered in the printhead before being extruded onto the substrate surface (Figure 1). Upon cooling the recognition motifs are able to reassemble, yielding a mechanically robust object. Furthermore, polyurethanes often afford flexible materials which may be of benefit for patient comfort and welfare in addition to possibly being desirable in the method of deployment.

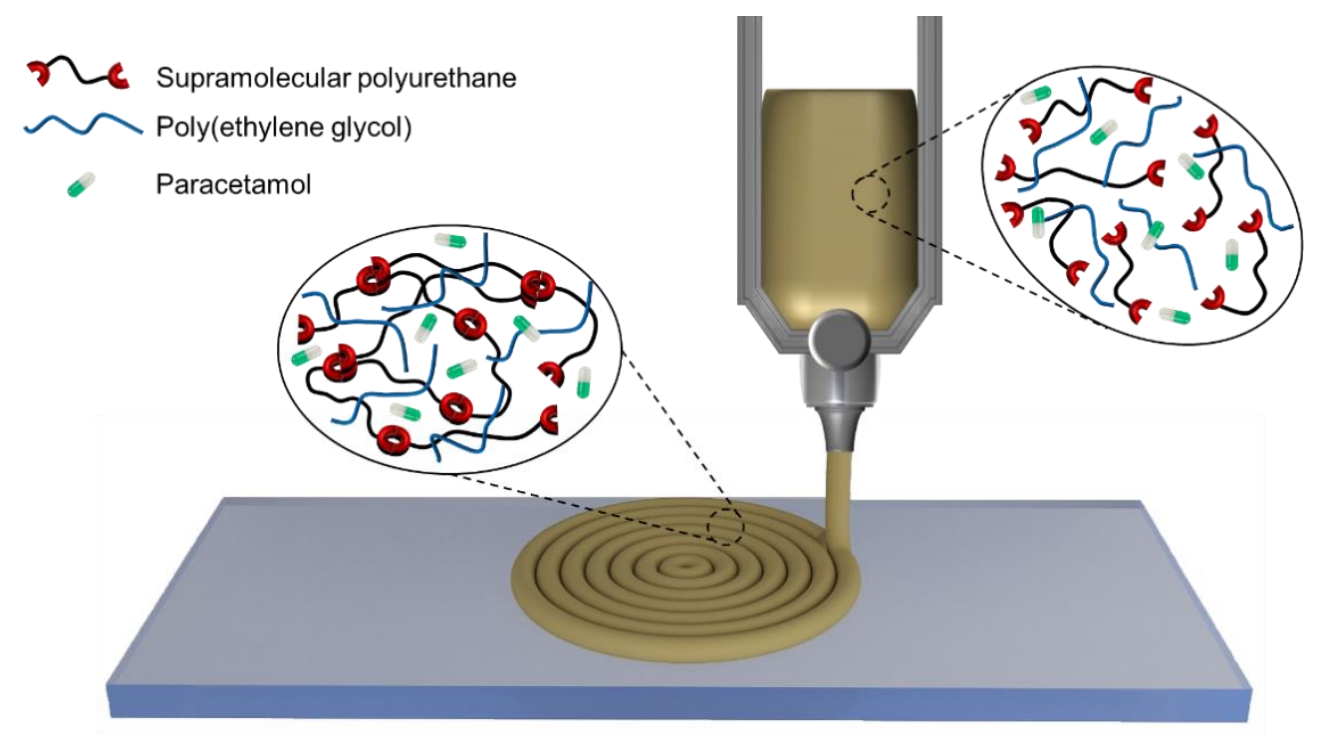

Figure 1: Schematic representation of the 3D printing of the prototype drug-release implant comprising of a dynamic self-assembling polyurethane, PEG and model drug (paracetamol).

\section{Polymer Synthesis}

To realise an implantable and potentially personalisable drug delivery device, an appropriate supramolecular recognition motif was required. To this end, a hydrogen bonding and $\pi$ - $\pi$ stacking end- 
group, namely $N$-(4-methoxybenzyl)-1-(4-nitrophenyl)methanamine ${ }^{31} \mathbf{1}$ was identified as a suitable assembly motif and was synthesised (Scheme S1) via the formation of the imine and subsequent reductive amination (see Figure S1 and Figure S2 for characterisation). To prepare the supramolecular polymer, an apolar hydrogenated polybutadiene (Krasol HLBH-P 2000, molecular weight as received $=2 \mathrm{~kg} \mathrm{~mol}^{-1}$ ) polyol 2 as a viscous liquid was end-capped with $4,4^{\prime}-\mathrm{MDI} 3$ at $80{ }^{\circ} \mathrm{C}$ in the bulk $^{38,39}$ to afford the pre-polymer 4 which was then terminated in solution (THF) by the recognition motif 1 (Scheme 1). The purified supramolecular polyurethane SPU1 was then afforded by multiple precipitations into methanol from THF. To confirm the successful synthesis of SPU1, NMR spectroscopy was undertaken (Figures S3-S5). ${ }^{1} \mathrm{H}$ NMR spectroscopy (Figure S3) revealed the appearance of the characteristic methylene resonances adjacent to the newly formed urethane (4.15 $\mathrm{ppm}$ ) and urea (4.70 and $4.45 \mathrm{ppm}$ ) moieties. In addition, urea and urethane carbonyl resonances were observed at $159.0 \mathrm{ppm}$ and $156.0 \mathrm{ppm}$ by ${ }^{13} \mathrm{C}$ NMR spectroscopy (Figure S4). IR spectroscopic analysis (Figure S6) confirmed the disappearance of the characteristic isocyanate stretch at $2340 \mathrm{~cm}^{-1}$ and the appearance of carbonyl stretches at 1708 and $1646 \mathrm{~cm}^{-1}$ corresponding to the newly formed urethane and urea bonds, respectively. Additionally, N-O stretches were observed at $1512 \mathrm{~cm}^{-1}$ and $1462 \mathrm{~cm}^{-1}$, assigned to the nitro group in the self-assembly motif and thus further confirming the incorporation of the end-group in the supramolecular polyurethane. Analysis of SPU1 by GPC (Figure S7) revealed a bimodal distribution, attributed to chain extension within the polyurethane, with a $M_{\mathrm{n}}$ of $6100 \mathrm{Da}$ and $Ð=2.06$, corresponding to approximately 2 repeat units per polymer chain. ${ }^{34}$ This is in close agreement with the integration analysis carried out by ${ }^{1} \mathrm{H}$ NMR spectroscopy. The thermal characteristics of the polymer were also probed using differential scanning calorimetry (Figure S8) which revealed a $T_{\mathrm{g}}$ at $-47.8^{\circ} \mathrm{C}$, which can be attributed to the soft domain of the phase separated supramolecular polymer, comprising of the hydrogenated polybutadiene. A second, less pronounced, $T_{\mathrm{g}}$ was observed at $19.45{ }^{\circ} \mathrm{C}$ which relates to the hard domains formed from hydrogen bonding motifs. These results are in good agreement with structurally related SPUs which demonstrate two thermal transitions. ${ }^{31,32,34}$ Furthermore, the temperature range of $T_{\mathrm{g}} \mathrm{S}$ of the bulk and self-assembled components indicated that the polymer was relatively malleable at room temperature and appropriate for use as a biomedical device produced by hot-melt extrusion printing. 


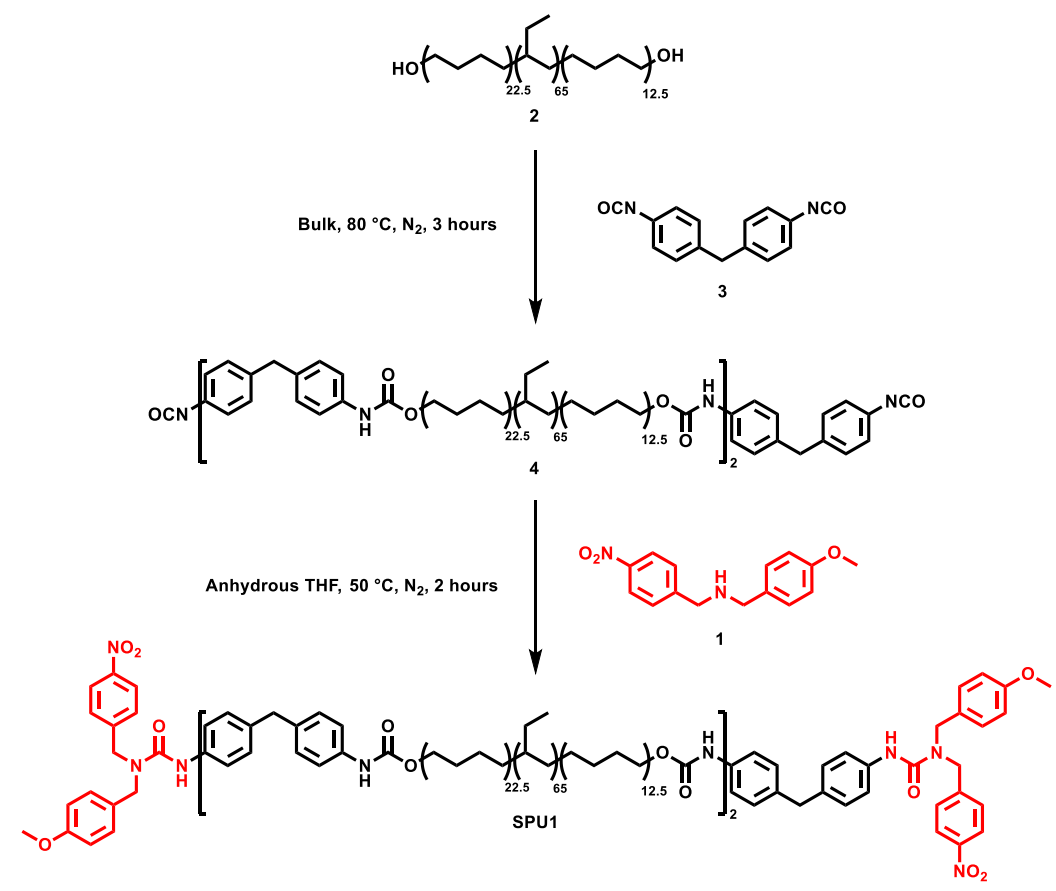

Scheme 1: Synthesis of the supramolecular polyurethane SPU1 by chain-extension of the polyol 2 with a diisocyanate 3 to yield the pre-polymer $\mathbf{4}$ which was subsequently terminated with recognition motif $\mathbf{1}$.

In order to assess the suitability of the supramolecular polymer for use in a biomedical device, films were required for analysis prior to $3 \mathrm{D}$ printing. To achieve this, the polymer was dissolved in the minimum amount of THF before drop casting onto PTFE moulds. The solution was left to dry under ambient conditions for 24 hours before drying under vacuum at $80{ }^{\circ} \mathrm{C}$ for a further 48 hours. Critically, SPU1 afforded a malleable elastomeric material (Figure S9) in contrast to the viscous hydrogenated polybutadiene precursor $\mathbf{2}$ as a result of the self-assembly of hydrogen bonding motifs and phase separation. ${ }^{40-44}$

To explore the thermal responsive nature of the supramolecular polyurethanes, variable temperature IR spectroscopic analysis (VT-FTIR) was conducted (Figure S10) between 20 and $160{ }^{\circ} \mathrm{C} .45,46$ A strong absorbance for both the free $\left(1730 \mathrm{~cm}^{-1}\right)$ and hydrogen-bonded $\left(1710 \mathrm{~cm}^{-1}\right)$ carbonyls units relating to urethane groups was observed, in addition to the ordered hydrogen bonded urea carbonyl moieties $\left(1655 \mathrm{~cm}^{-1}\right)$ and bound $\mathrm{NH}$ groups $\left(3313 \mathrm{~cm}^{-1}\right)$ in SPU1 at room temperature. Indeed, the intensity of the urethane, urea and $\mathrm{NH}$ absorbance bands were seen to weaken and/or shift with increased temperature, ${ }^{38}$ as a result of dissociation of the hydrogen bonds which was consummate with a new absorbance at $3440 \mathrm{~cm}^{-1}$, indicative of free NH bonds. Most notably, the absorbance corresponding to hydrogen bonded urethanes could be seen to diminish and coalesce with the adjacent free carbonyl signal. To further quantify the disruption of the hydrogen bonding network at elevated temperatures, the area of the hydrogen bonding urethane carbonyls and $\mathrm{NH}$ absorptions were calculated (Figure S11) by deconvoluting and fitting the absorbance bands. Over the temperature regime investigated, hydrogen 
bonded urethane carbonyls demonstrated a $c a .40 \%$ reduction in abundance, whilst the prevalence of free urethane carbonyls increased by $c a .40 \%$. Additionally, a decrease of $c a .45 \%$ in absorbance area was observed in hydrogen bonding NHs. Additionally, upon cooling, the intensity of signals relating to hydrogen bonding motifs were fully restored, demonstrating the thermally dynamic character of the polymer network.

\section{Biocompatibility Studies}

To fabricate a safe drug delivery device, it is essential that the polymer be biocompatible. It has been reported $^{47}$ that polyurethanes are biocompatible, and as a consequence have been utilised in designing drug delivery systems. Moreover, we have demonstrated that a structurally related polyurethane with morpholine end groups has shown biocompatibility with human fibroblasts. ${ }^{32}$ In the current system where the ratio of the end group to the total polymer did not exceed $(8 \mathrm{~mol} \%)$, the terminal groups have been modified in order to obtain a polymeric material with desirable physical characteristics to enable it to be used in hot-melt extrusion printing. As a first step in establishing the potential of SPU1 in this regard, the cytotoxicity of the polyurethane films was evaluated. Liquid extracts from the polymeric films were used to determine the biocompatibility of the polymeric films by an MTT assay. To achieve this, mouse fibroblasts (L929), immortalised cells used routinely to evaluate biocompatibility, were exposed to the liquid extracts. The polyurethane was found to be non-toxic, as cell viability was in excess of $94 \%$ after exposure to undiluted (100\%) liquid extracts from the polymer (Figure 2). Importantly this is not significantly different from the negative control and hence SPU1 was a potential candidate for 3D printing of a biomedical device. Interestingly, the exchange of the end groups, from morpholine to $\mathrm{N}$-(4-methoxybenzyl)-1-(4-nitrophenyl)methanamine resulted in a non-cytotoxic polymer. These results are in good agreement with our previously reported data, in which a poly(caprolactone)-derived supramolecular polyurethane with the analogues $\mathrm{N}$-(4-methoxybenzyl)-1(4 nitrophenyl)methanamine end groups was found to be non-toxic. ${ }^{48}$ 


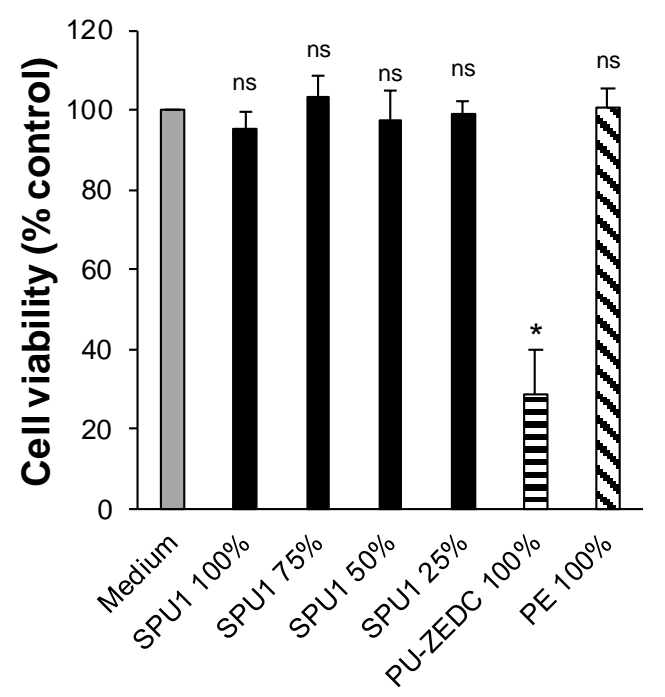

Figure 2: Cytotoxicity of liquid extracts from the Medium, supramolecular polyurethane (SPU1) at different dilutions $(100 \%, 75 \%, 50 \%, 25 \%)$, positive control (PU-ZEDC) and negative control (PE), both at 100\%. Data are presented as mean \pm s.e.m. Statistical significance with respect to untreated cells (medium) was determined by ANOVA followed by Bonferroni post hoc test ( $*$ TBC $-\mathrm{P}<0.05$; ns = non-significant).

\section{Polymer Formulations and Analysis}

In order to formulate a drug delivery device with effective rates of release in addition to good processability, a formulation was developed comprising of the supramolecular polyurethane and PEG. A commercially available PEG $\left(M_{\mathrm{w}}\right.$ as received $\left.=20 \mathrm{~kg} \mathrm{~mol}^{-1}\right)$ was selected as an excipient to modify the rate of release of the active from the formulation and further tune the extrusion characteristics of the polymer blend. ${ }^{35}$ Our previous studies have revealed that less than $8 \mathrm{wt} \%$ of PEG is able to modify the release rate of a dosage form whilst also having a positive contribution to the printability of the formulation. ${ }^{49}$ Paracetamol was selected as the model drug and formulated at $16 \mathrm{wt} \%$. We have previously used paracetamol in a number of oral-dosage form 3D printing studies, illustrating an ability to achieve formulations that meet Pharmacopeia standards of manufacture, e.g. weight uniformity, hardness, friability etc. ${ }^{50,51}$

Formulations for 3D printing were prepared by blending SPU1 and PEG $\left(20 \mathrm{~kg} \mathrm{~mol}^{-1}\right)$ in ethyl acetate $\left(0.15 \mathrm{~mL} \mathrm{~g}^{-1}\right)$ prior to the addition of paracetamol to yield a homogeneous mixture (Table 1) before removing the solvent in vacuo.

Table 1: Composition of the formulations F1 and F2.

\begin{tabular}{cccc}
\hline & Polyurethane (wt\%) & PEG (wt\%) & Paracetamol (wt\%) \\
\hline F1 & 80.00 & 4.00 & 16.00 \\
F2 & 76.00 & 8.00 & 16.00
\end{tabular}


In order to probe the effect of PEG on the thermoresponsive supramolecular polyurethane, VT-FTIR spectroscopic analysis was conducted between $20^{\circ} \mathrm{C}$ to $160^{\circ} \mathrm{C}$ (Figure S10). The formulations $\mathbf{F 1}$ and F2 demonstrated significant decreases in absorbances associated with hydrogen bonding (bound $\mathrm{NH}$ $3313 \mathrm{~cm}^{-1}$ and urethane carbonyls $\left.-1710 \mathrm{~cm}^{-1}\right)$ and a concurrent increase in free urethane $\left(1740 \mathrm{~cm}^{-1}\right)$ and free $\mathrm{NH}\left(3440 \mathrm{~cm}^{-1}\right)$ absorbances. Additionally, the stretch at $1655 \mathrm{~cm}^{-1}$, corresponding to ordered hydrogen bonded urea carbonyl units $\left(1655 \mathrm{~cm}^{-1}\right)$ proceeded to shift to a higher wavenumber $\left(1670 \mathrm{~cm}^{-}\right.$ ${ }^{1}$ ) upon heating, indicating dissociation of hydrogen bonding of the urea moieties. Whilst the rate of dissociation of hydrogen bonding is approximately equivalent in SPU1, F1 and F2 (Figure S11), the composition of hydrogen bonding moieties differs between the neat polyurethane and the formulation. The hydrogen bonded NH moieties remained the predominant relative to the carbonyl moieties, however, the relative prevalence of hydrogen bonding carbonyls was observed to have decreased in F1 and $\mathbf{F} 2$ when compared to SPU1.

To study the physical properties of the polymer formulations as related to assessing their suitability for printing and potential long-term stability as implants, films were prepared as previously described with the addition of the relevant weight percentage of PEG. The polymer films were then annealed in the oven for 72 hours at $80^{\circ} \mathrm{C}$ until dry. To assess the thermal characteristics of the polyurethane SPU1 and formulations $\mathbf{F 1}$ and $\mathbf{F} 2$ and rheological analysis of film was undertaken (Figure 3). In all instances, the physical properties of the polymer were governed by the elastic character of the materials, in the low temperature regime $\left(c a .0-35^{\circ} \mathrm{C}\right)$, as demonstrated by the storage modulus ( $\left.\mathrm{G}^{\prime}\right)$ being in excess of the loss modulus (G”). Upon further heating, G' and G" gradually decreased as a result of the dissociation of the supramolecular polyurethane network and shift in equilibrium towards the unbound state. In contrast, the rheological behaviour of the hydrogenated polybutadiene $\mathbf{2}$ is dominated by the viscous character of the polymer through all temperature regimes, as exemplified by a constant phase angle of $c a .90^{\circ}$ upon heating (Figure S12). When considering SPU1 alone (Figure 3a), the phase angle is initially less than $45^{\circ}$ at low temperatures, indicating the material properties are predominantly elastic in nature. At $38^{\circ} \mathrm{C}$, the phase angle crosses $45^{\circ}$ and therefore viscous behaviour governs the properties of polyurethane at temperatures in excess of this. By increasing the PEG content, the temperature at which the cross-over between G' and G" occurred was observed to increase as a result of the PEG excipient retarding the transition from elastic to viscous behaviour within the system (Figure $3 \mathrm{~b}$ and 3c). Therefore, the incorporation of PEG in the formulation increased the temperature of the cross-over point, shifting this away from core body temperature $\left(37^{\circ} \mathrm{C}\right)$. In doing so, this may contribute to the control mechanism of drug release using the PEG as well as making the resulting implant more mechanically robust under normal operating temperatures within the body. 

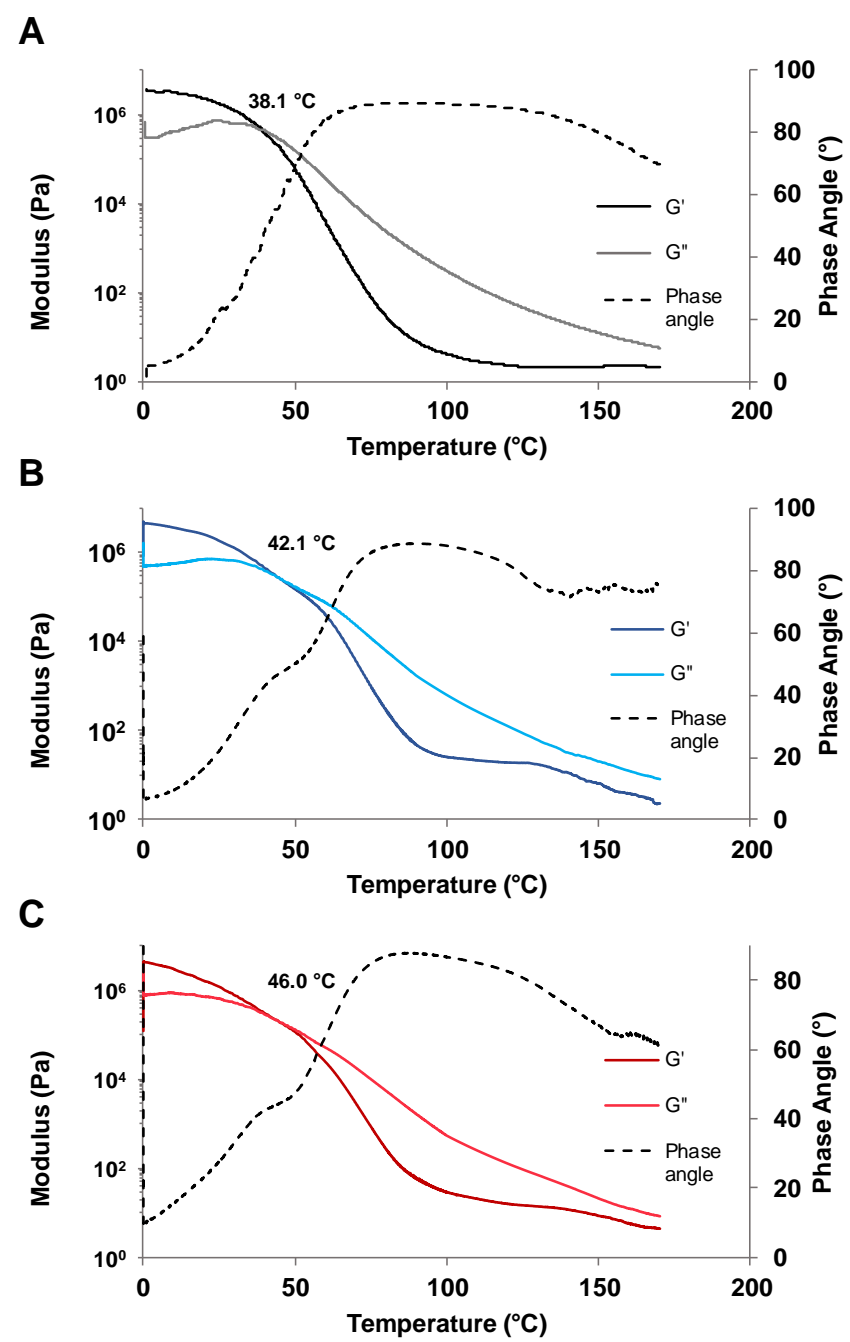

Figure 3: Rheological behaviour of A) SPU1, B) F1 and C) F2 with respect to temperature.

To further explore the morphology of the supramolecular polyurethane $\mathbf{1}$ and the phase behaviour of the PEG in Formulations F1 and F2, SAXS analysis was conducted (Figure S13). SPU1 exhibits typical micro-phase separation behaviour as confirmed by the Bragg peak centred at $73.6 \AA$ which arises from immiscibility of the polar hard (self-assembling) and apolar soft (polymer backbone) domains. PEG displayed Bragg peaks at $154.6 \AA$ and $84.9 \AA$, respectively whilst the addition of PEG to the supramolecular polyurethane to yield formulations $\mathbf{F 1}$ and $\mathbf{F} 2$ for 3D printing resulted in shifts to lower domain spacing $(\mathbf{F 1}=66.9 \AA, \mathbf{F 2}=69.3 \AA$ ). Interestingly, no apparent diffraction peaks attributed to PEG were observed in the formulations. This data suggests that the PEG within the formulation was thoroughly mixed into the phase separated polyurethane architecture through incorporation into the polar hard domains. Furthermore, this data is supported by VT-FTIR spectroscopy (Figure S10 and Figure S11) which suggests that PEG is incorporated into the self-assembling polyurethane matrix as demonstrated by weakening of the hydrogen bonding carbonyl absorbances relative to the neat SPU1. 
Tensile testing was carried out to further understand the mechanical characteristics of the polymer formulations (Figure 4). From the cast films, strips of polymer ( $c a .40 \mathrm{~mm} \times 5 \mathrm{~mm} \times 1 \mathrm{~mm}$ ) were produced for analysis, by extended to failure, the corresponding mechanical properties were calculated from the individual stress-strain curves (Table 2).

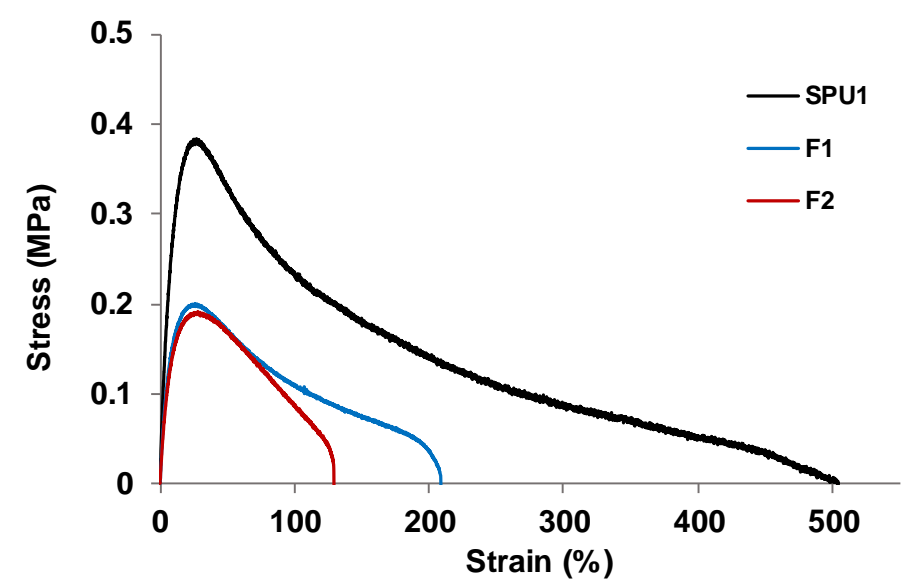

Figure 4: Stress/strain curves for drop-cast films of the supramolecular polyurethane (SPU1) and formulations for 3D printing (F1 \& F2) as cast from solution.

The supramolecular polyurethane exhibited the greatest ultimate tensile strength (0.37 MPa), Young's modulus (2.34 MPa), modulus of toughness $(0.67 \mathrm{MPa})$ and elongation at break (500\%). In agreement with rheological studies, the incorporation of $4 \mathrm{wt} \%$ PEG to the formulation (F1) dramatically reduced the mechanical properties of the formulation. A loss of approximately $50 \%$ of the ultimate tensile strength $(0.20 \mathrm{MPa}), 20 \%$ of the Young's modulus $(1.87 \mathrm{MPa})$ and $69 \%$ of the modulus of toughness $(0.21 \mathrm{MPa})$ was observed. Furthermore, the elongation at break was observed to have decreased to $210 \%$, a reduction of $290 \%$ when compared to SPU1. The decrease in mechanical performance was attributed to the PEG excipient interrupting the hydrogen bonding network and thus reducing selfassembly between the hydrogen bonding motifs. This was corroborated by SAXS data (Figure S13) collected from films of the formulation which suggested that PEG was thoroughly mixed into the phase separated polymer matrix, signified by the omission of diffraction peaks relating to PEG. It is likely that the polar PEG molecules would have migrated to the polar self-assembling hard domains, thus interrupting the self-assembly. Similar reductions in mechanical performance were also observed in F2. Whilst a reduction in the mechanical properties were observed through the addition of PEG to the supramolecular polymer matrix, the resulting properties were still within the printable parameters range for hot-melt extrusion 3D printing of a geometrically well-defined object that could be considered for future development into an implant and therefore this material was utilised in the development of test structures. 
Table 2: Tensile data for the neat supramolecular polymer (SPU1) and formulations containing PEG (F1 \& F2).

\begin{tabular}{cccc}
\hline & $\begin{array}{c}\text { Ultimate Tensile } \\
\text { Strength (MPa) }\end{array}$ & $\begin{array}{c}\text { Youngs Modulus } \\
(\mathbf{M P a})\end{array}$ & $\begin{array}{c}\text { Modulus of } \\
\text { Toughness (MPa) }\end{array}$ \\
\hline SPU1 & $0.37 \pm 0.02$ & $2.34 \pm 0.27$ & $0.67 \pm 0.05$ \\
F1 (4\% PEG) & $0.20 \pm 0.01$ & $1.87 \pm 0.10$ & $0.21 \pm 0.08$ \\
F2 (8\% PEG) & $0.20 \pm 0.03$ & $1.52 \pm 0.13$ & $0.24 \pm 0.06$ \\
\hline
\end{tabular}

\section{D Printing and Analysis of Prototype Implants}

To produce a prototype implant, a bar geometry $(30 \mathrm{~mm} \times 3 \mathrm{~mm} \times 2.5 \mathrm{~mm})$, similar to that used for some contraceptive implants, was designed using computer aided design (CAD) before being converted into layers ready for $3 \mathrm{D}$ printing. The formulations were loaded into an aluminium extrusion printing cartridge and placed in a Cellink INKREDIBLE printer. The formulations were subsequently deposited with control over parameters such as extrusion pressure, temperature, printing layer height, printing speed and material infill (see Supplementary Videos 1 and 2). Bar samples were then printed to evaluate the spatial definition and composition of the materials (Figure 5). Drug-free formulations were also printed as controls using the same conditions for materials testing.
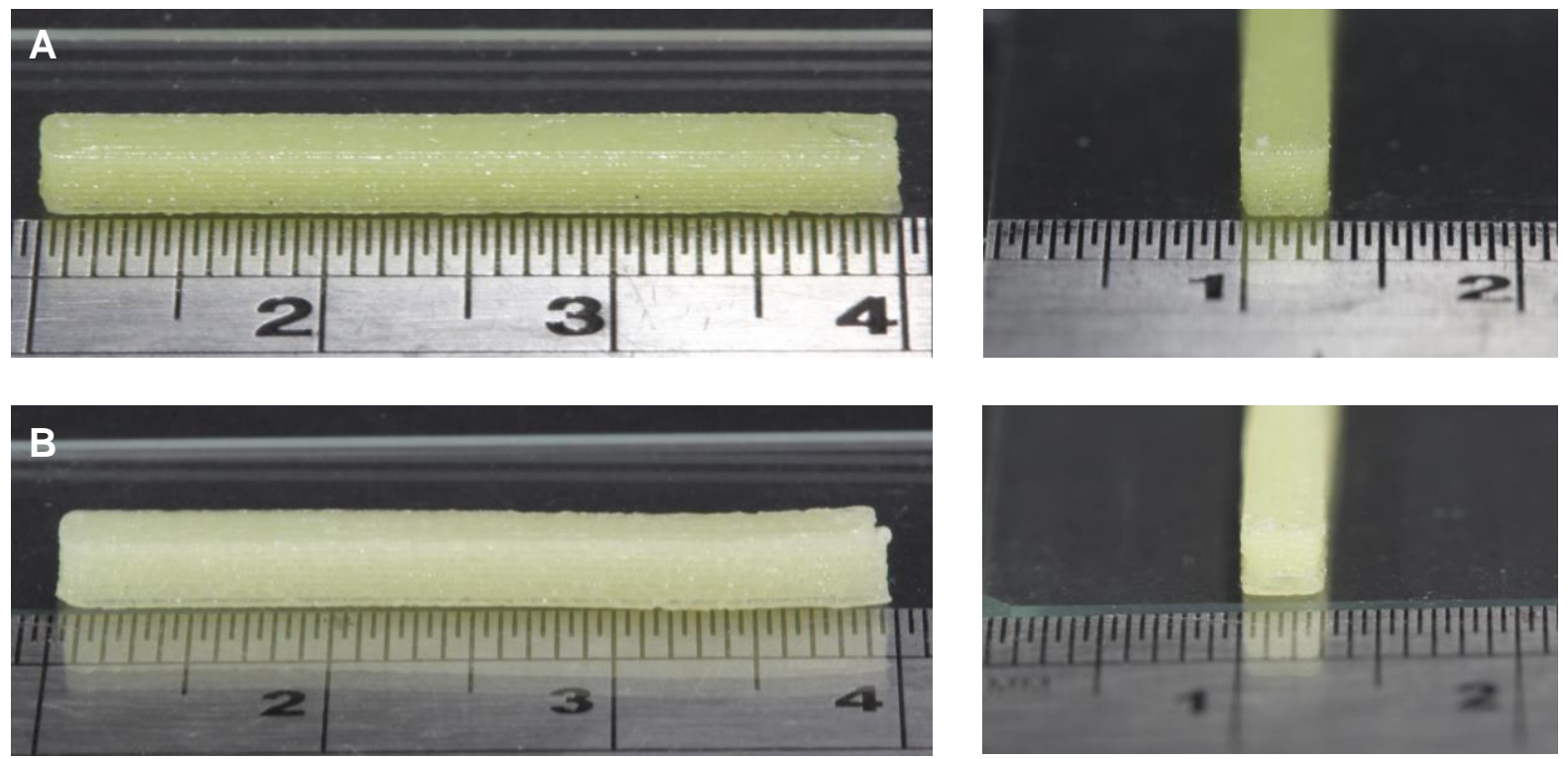

Figure 5: Side-on and end-on view images of the 3D printed bars of A: F1 (4\% PEG) and B: F2 (8\% PEG).

The dimensions and masses of the prototype implants $(N=10$ bars $)$ were then analysed (Table 3$)$ to assess the resolution and reproducibility of the printing process. Less than $2 \%$ deviation was observed 
in the length and thickness for both F1 and F2 (Table S1), indicating good printability of the polymer formulations. The widths of the printed bars were also analysed and were revealed to be slightly more variable $(\mathbf{F 1}= \pm 4 \%, \mathbf{F} 2= \pm 3 \%)$, however, this still demonstrated good reproducibility of the printed prototype implants. The weight of individual printed bars was also measured (Table S2) and analysis showed that the printed implants comprised of F1 comply with the United States Pharmacopeia (USP) specification ( $\pm 7.5 \%$ deviation) ${ }^{52}$ Larger variations up to $9.5 \%$ were noted for the bars printed from $\mathbf{F 2}$ which may relate to the increased PEG content and its effect on printability, but this remains to be determined.

Table 3. A summary of the dimensions, print parameters and masses of printed bar implants $(\mathrm{n}=10$ for all measurements).

\begin{tabular}{ccc}
\hline & F1 & F2 \\
\hline 3D model geometry & \multicolumn{2}{c}{ Bar } \\
3D model dimension (mm) & $30(\mathrm{~L}) \times 3(\mathrm{w}) \times 2.5(\mathrm{H})$ \\
Length (L)* & $29.58 \pm 0.19$ & $29.59 \pm 0.14$ \\
Width (W)* & $3.11 \pm 0.06$ & $3.17 \pm 0.03$ \\
Thickness (H)* & $2.54 \pm 0.02$ & $2.71 \pm 0.01$ \\
Mass (g)* & $0.224 \pm 0.0036$ & $0.212 \pm 0.0108$ \\
\hline
\end{tabular}

To confirm the incorporation of the drug in the formulation and assess its stability during the printing process, IR spectroscopy (Figure 6a) was conducted on the pure components (SPU1, PEG, and paracetamol) and the printed formulations (F1 and F2). From the cast film of SPU1, both urethane $\left(1708 \mathrm{~cm}^{-1}\right)$ and urea $\left(1644 \mathrm{~cm}^{-1}\right)$ carbonyl stretches were observed.. ${ }^{31}$ Sharp absorptions at $1521 \mathrm{~cm}^{-1}$ and $1462 \mathrm{~cm}^{-1}$ were attributed to the $\mathrm{N}-\mathrm{O}$ stretch arising from the nitro functionalised aryl group of the terminal self-assembly motif in the polymer. A characteristic N-H stretch from the urea was also identified at $3323 \mathrm{~cm}^{-1}$, and N-H plane deformation and C-H stretch were identified at $1523 \mathrm{~cm}^{-1}$ and $2921 \mathrm{~cm}^{-1}$, respectively. An out of plane C-H bend was also observed around $815 \mathrm{~cm}^{-1}$. Two strong absorptions, centred at $2882 \mathrm{~cm}^{-1}$ and $1096 \mathrm{~cm}^{-1}$ were observed in PEG and were attributed to C-H stretching and the $\mathrm{C}-\mathrm{O}$ stretching within the polymer, respectively. ${ }^{53}$ Finally, an N-H stretch $\left(3323 \mathrm{~cm}^{-1}\right)$, out of plane $\mathrm{C}-\mathrm{H}$ bend $\left(836 \mathrm{~cm}^{-1}\right)$, carbonyl adsorption bands $\left(1650 \mathrm{~cm}^{-1}\right)$ and N-H plane deformation at $1561 \mathrm{~cm}^{-1}$ were observed in paracetamol in addition to the expected O-H stretch at 3161 $\mathrm{cm}^{-1} \cdot{ }^{54}$

IR spectroscopy of the 3D printed formulations (F1 and F2) were then analysed to assess the stability of the components. Indeed, N-H stretches were observed at $3323 \mathrm{~cm}^{-1}$ for $\mathbf{F 1}$ and $3315 \mathrm{~cm}^{-1}$ for $\mathbf{F 2}$, comparable to that seen in pure SPU1 and paracetamol. A carbonyl stretch was observed at $1654 \mathrm{~cm}^{-1}$ in both $\mathbf{F 1}$ and $\mathbf{F} 2$ relating to paracetamol in addition to an adsorption at $1709 \mathrm{~cm}^{-1}$ which was attributed to the carbonyl moiety from SPU1. Additionally, N-O stretches were observed at $1516 \mathrm{~cm}^{-1}$ and 1462 $\mathrm{cm}^{-1}$, further confirming the presence of the supramolecular polymer in formulations $\mathbf{F} 1$ and $\mathbf{F} 2$. The 
N-H plane deformation peaks from both paracetamol and SPU1 overlap and can be found at $1514 \mathrm{~cm}^{-1}$ in both F1 and F2. The O-H stretch of paracetamol was not apparent in F1 and F2, possibly as a result of hydrogen bonding between the active and the polymer matrix.

Powder X-ray diffraction (PXRD) of the pure components and the two printed formulations was also performed in order to examine the state of the dispersed drug in the final formulations (Figure 6b). The SPU1 diffraction pattern exhibits a broad halo, indicating that the polymer is amorphous. This is in accordance with data previously reported by Feula et al. ${ }^{17}$ In contrast, the diffraction pattern of the PEG excipient suggests that this material is at least in-part crystalline, as indicated by the two broad diffraction signals. ${ }^{31}$ The PXRD pattern of pure paracetamol matches that of crystalline form I previously reported..$^{55}$ After formulation and printing, there is no evidence of a phase transformation of paracetamol in the formulations, F1 and F2. Owing to the low percentage composition of PEG in F1 and F2, nothing definitive can be said about the crystallinity of PEG in these formulations after printing, based on the PXRD data alone. However, as there is no evidence of any change in the crystalline form of the paracetamol in the printed formulations $\mathbf{F 1}$ and $\mathbf{F 2}$, this suggests that the printing process does not alter or damage the constituent components of the formulations. This was further studied by DSC analysis of SPU1, F1 and F2 (Figure S8). Two glass transitions at $c a .-47^{\circ} \mathrm{C}$ and $c a .0{ }^{\circ} \mathrm{C}$, typical of phase separated polyurethane are noted. ${ }^{33,56}$ Although, no meaningful changes in thermal transition temperatures were observed, a less intense melting peak at $c a .57{ }^{\circ} \mathrm{C}$ that corresponds to PEG was present in the thermograms of the printed formulations. These results suggest that the high shear and temperature experienced by the formulation in the hot-melt extrusion 3D printing process lead to a more efficient blending of SPU1 and PEG and therefore, a loss in PEG crystallinity.

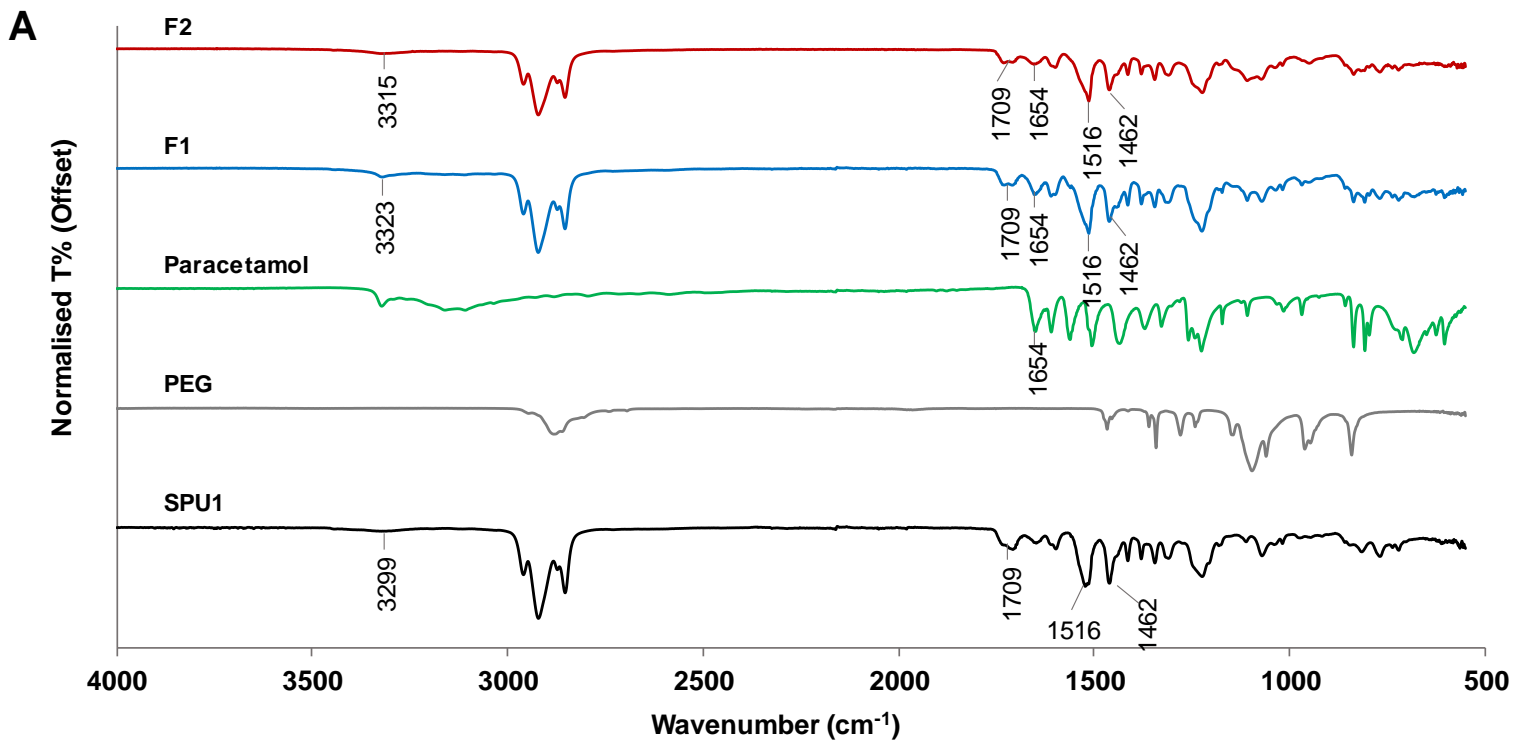




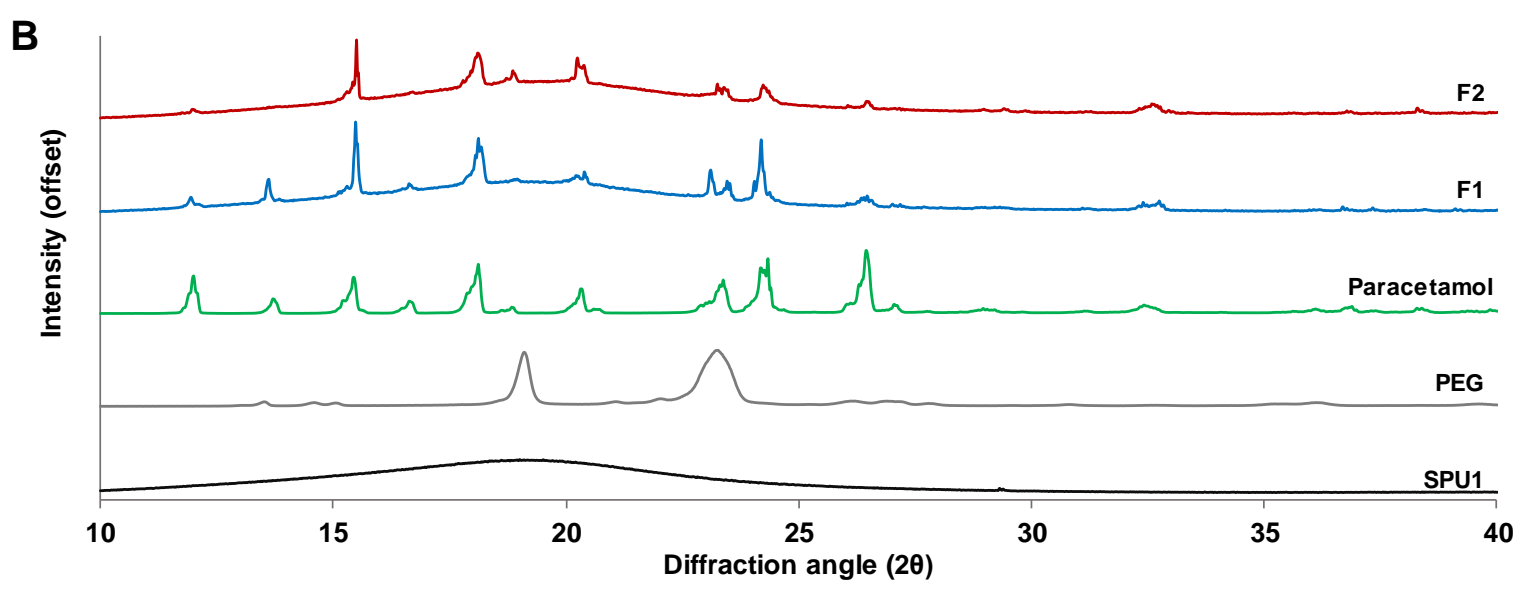

Figure 6: A) FTIR spectra and B) PXRD patterns of pure SPU1, PEG $20 \mathrm{~kg} \mathrm{~mol}^{-1}$, paracetamol and the printed bars of $\mathbf{F} 1$ and $\mathbf{F} 2$.

To explore the effect of 3D printing on the formulation, additional SAXS experiments were conducted. Scattering patters were collected of the printed formulations, F1 and F2, and compared to the cast analogues (Figure S14). A decrease in domain sizes ( $c a .4 \AA$ A shift) was observed in both formulations as a consequence of the $3 \mathrm{D}$ printing process. Although a small change, this may be as a result of the thermal and shear processes associated with 3D hot-melt extrusion printing annealing the polymer formulation and reducing the size of the domains though enhanced mixing of the two polymer phases.

It was noted that after a week, the surface of the printed prototype implant appeared to smoothen slightly (Figure S15), and visually the layers appeared to become more homogeneous as a result of creep. To monitor the effects of creep on the mechanical properties of drug-free formulations, 3D printed samples were aged under ambient conditions for 7 days. To study the effect on rheological behaviour, a frequency sweep at $25{ }^{\circ} \mathrm{C}$ was performed in the linear viscoelastic region and between $0.01 \mathrm{~Hz}$ and $100 \mathrm{~Hz}$ (Figure S16). It was noted that the rheological properties did not show any significant improvement when considering either processing technique. The rheological properties of the 3D printed samples over time were also compared with a sample prepared by drop-casting (Figure S17). Interestingly, whilst the drop cast films do appear to be a good model for the 3D printed disks, the printed objects exhibited a modest increase in modulus (cast $=4.5 \times 10^{4} \mathrm{~Pa}$, printed $=1 \times 10^{5} \mathrm{~Pa}$ ). It is believed that the elevated temperature, combined with high sheer forces imparted on the polar PEG and apolar SPU components of the formulation during printing resulted in a more intimate mixture of the polymers (and hence stiffer) when compared to the drop cast sample.

To further examine the effects of creep on the mechanical properties of the 3D printed drug-free formulations, the printed bars $(c a .40 \mathrm{~mm} \times 5 \mathrm{~mm} \times 0.7 \mathrm{~mm})$ were elongated to break, and stress/strain profiles recorded (Figure S18). Interestingly, the ultimate tensile strength (Figure S19a) of the printed material was seen to reduce by approximately $20 \%$ in $\mathbf{F 1}$ and $45 \%$ in $\mathbf{F} 2$ when compared to the pure 
polymer film prepared by drop-casting (Table S1), indicating that 3D printing does not fully translate the materials ability to withstand loads as a result of voids and poor interlayer adhesion compared to the homogeneous drop-cast films. Furthermore, although interlayer contact appears to improve visually over time, the ultimate tensile strength remains approximately constant between printing and day 7 for both formulations (F1 and F2). The Young's modulus (Figure S19b) was approximately equivalent when comparing 3D printed and drop-cast data for formulations F1 and F2 (Table S3), revealing that along the axis of printing and elongation, the polymers properties were unaffected by formulation and processing. An upward trend was observed in Young's modulus over time which was attributed to the dynamic nature of the supramolecular polymer within the printed formulation. During the aging process, the printed layers undergo minor deformation as a consequence of thermal relaxion and reassembly of the supramolecular polymer in a lower energy regime. ${ }^{57}$ Thus, the printed objects which have undergone creep over time demonstrate a moderate increase in strength and are therefore more resistant to permanent deformation. This relationship was most pronounced in $\mathbf{F 1}$ where the percentage composition of PEG was at its lowest, allowing for better network reorientation over time.

Although a slight decrease in the resolution of the printed object may occur, the deformation may result in enhanced mechanical properties by virtue of improved interlayer adhesion and increased contact area between printed layers. Mechanical analysis of creep by rheology and tensile testing can be correlated with rheological analysis of the cast materials (SPU1, F1 and F2: Figure 3) where the temperature at which $G^{\prime}$ and $G^{\prime \prime}$ was observed to cross (phase angle $=45^{\circ}$ ) is close to ambient temperature in all instances, thus allowing the dynamic supramolecular polymer to disassemble and reassemble slowly over extended timeframes.

\section{Drug release from the Printed Prototype Implants}

The initial rate of paracetamol release from the printed formulations was determined with HPLC. (Figure 7). The printed implants were found to exhibit similar release rates, with up to $2.5 \%$ paracetamol and 2\% paracetamol were released for formulations, F1 and F2, respectively. With such low release levels fitting models to the such data to predict long-term release should be done with some caution. With this in mind, a first order release model was implemented to approximate the behaviour of the implants beyond 168 hours (Table S4). F1 and F2 are predicted to fully release the active over 8.5 months and 5 months, respectively. This is, at the very least, indicative that suitable timescales for applications of these materials as implants will be achievable and that composition variations, such as PEG amount as here, can be used to tune release rate. In order for the material to be suitable for implant applications, it should robustly retain its designed shape. It should be noted that deformation of the 
implants (Figure S20) was observed after dissolution testing; suggesting that further optimisation of the material may be needed.

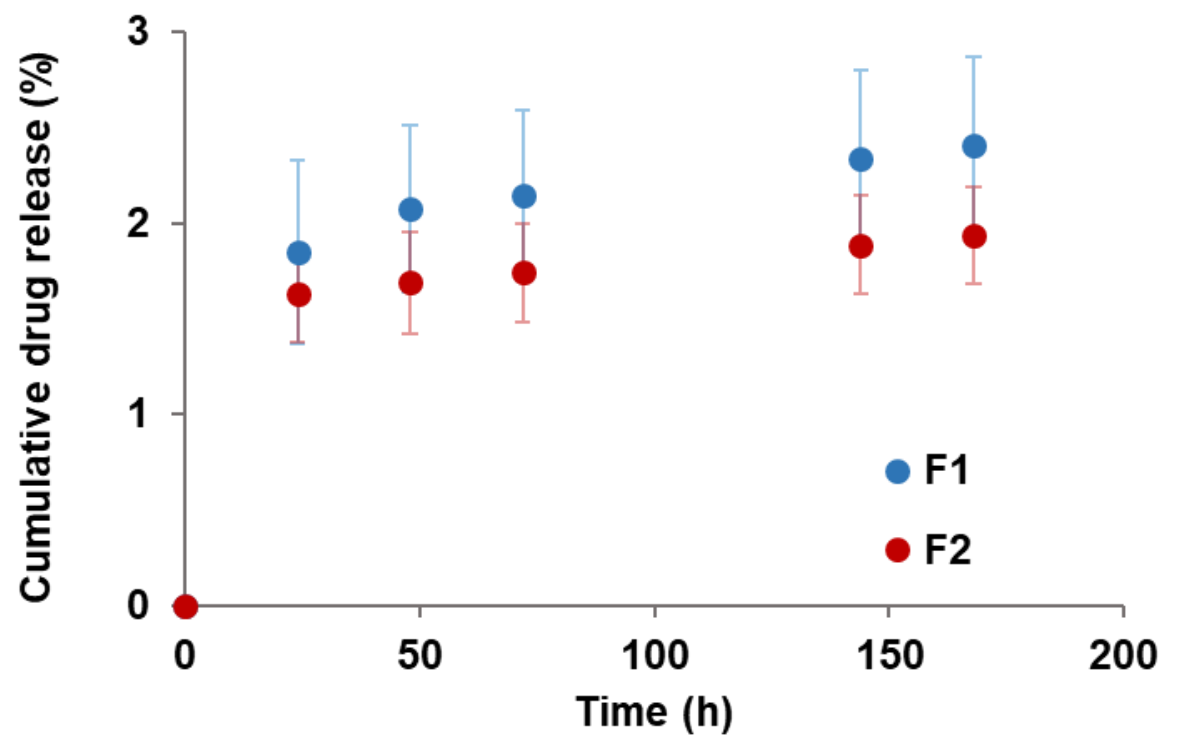

Figure 7: Release behaviour of paracetamol over 7 days from the printed formulations, F1 and F2. Data shown are average of 5 repetitions.

SEM was used to characterise the morphology of the printed formulations (F1 and F2). Hot melt extrusion of both formulations gave rise to homogeneous mixtures of SPU1 and PEG in addition to well dispersed paracetamol within the polyurethane matrix as demonstrated by the absence of agglomerates and crystallites. To examine the effects of drug release on morphology, analysis was also carried out after 7 days of dissolution studies. A stark contrast in the structure of the printed formulations was observed after treatment with the dissolution media (Figure 8), resulting in a micro-porous network which was attributed to the leaching of the PEG excipient. As expected, the pores were more prominent in the $\mathbf{F 2}$ as a consequence of the increased loading of PEG in the formulation. Clearly, the size of these pores cannot be the only contributing factor to drug release as on the time-scales observed in our dissolution studies there is not a direct correlation with this microscopic observation and drug release. Many factors are likely to contribute to drug release, including pore connectivity, pore size distribution and exposed surface area and these remain a matter for future formulation optimisation studies. Whether these features would affect the integrity of the implant over longer timescales should also be considered and may explain the deformation of the implants (Figure S20). 

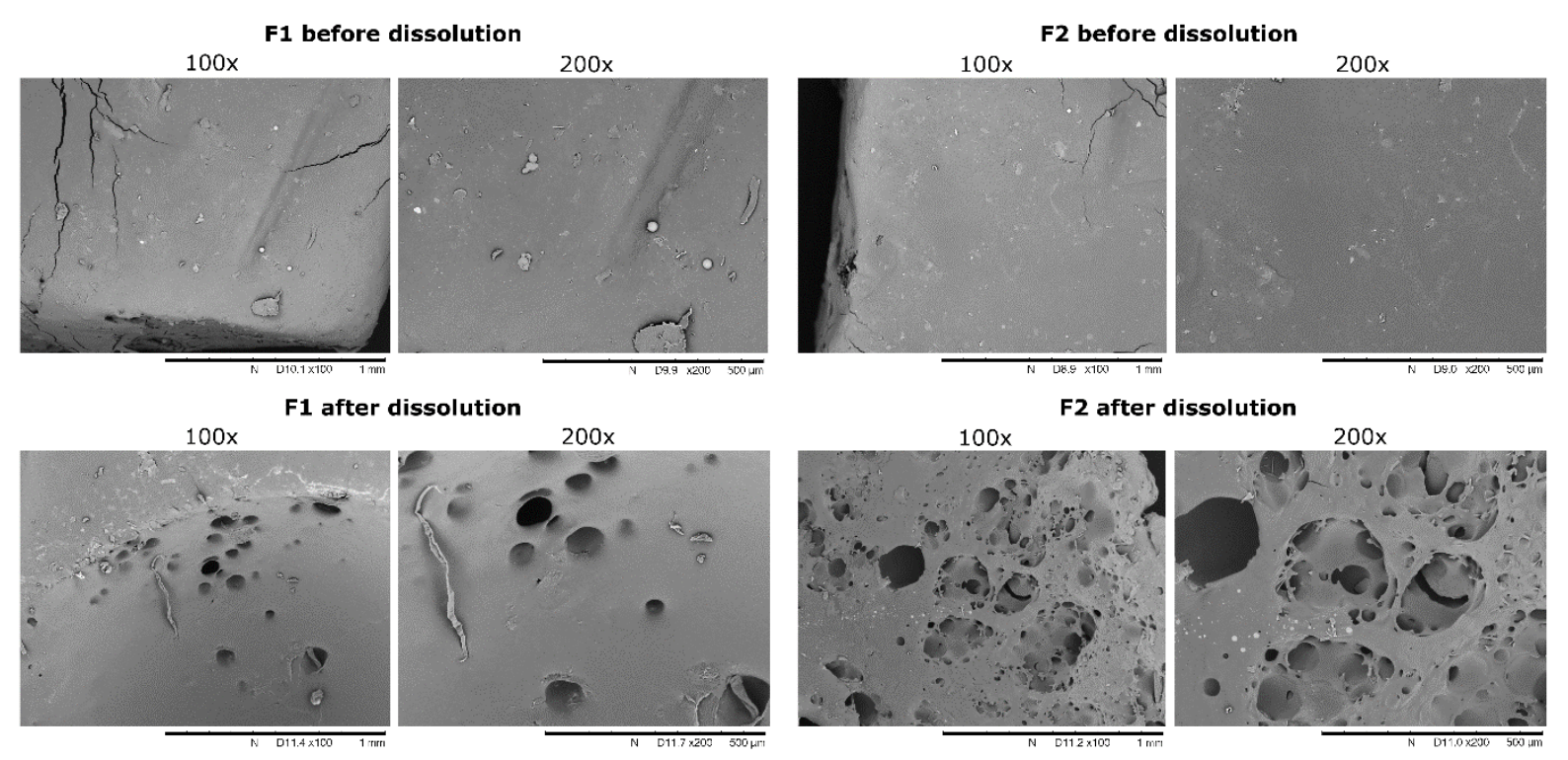

Figure 8: SEM images of sample cross-section before and after the dissolution tests.

\section{Conclusions}

Through molecular design, we have prepared a thermo-responsive supramolecular polyurethane able to dissemble and assemble at a specific transition temperature and evaluated its potential biocompatibility and cytotoxicity. The mechanical properties of the SPU1 showed that the material is self-supporting and stiff, yet flexible. The material was therefore investigated as a candidate for 3D printed implant applications. In order to investigate the release characteristics, paracetamol (16 wt $\%)$ was dispersed into the SPU1 formulated with $4 \mathrm{wt} \%$ or $8 \mathrm{wt} \%$ PEG excipients to investigate the ability to manipulate drug release profile. Bar implants were then designed, and the formulations successfully 3D printed using a hot-melt extrusion printer from melt at $100{ }^{\circ} \mathrm{C}$.

The mechanical characteristics of the formulations were analysed which indicated that the material was a suitable matrix for hot-melt extrusion printing. The paracetamol in the printed implants was identified as form 1 by PXRD and there were no adverse effects observed in printing the active in the formulations after extrusion. HPLC was used to quantify drug release. From initial dissolution experiments, the release profiles were fit to a first-order drug release model. This approximation indicates that $36 \mathrm{mg}$ of paracetamol would be released within upto 8.5 months depending on PEG loading. This slow and potentially controllable release profile is consistent with timescales desired and needs for optimisation for drug eluting implants. However, we note that deformation of the implants over course of dissolution characterization. Finally, SEM was used to examine the morphology of the printed implants before and after drug release. The transition temperatures of the SPU may need to be further considered and optimised for long term release applications. 


\section{Experimental}

\section{Materials}

All reagents were purchased from Sigma Aldrich or Alfa Aesar and used as received with the exception of Krasol HLBH-P2000 $\left(M_{\mathrm{w}}\right.$ as received $\left.=2 \mathrm{~kg} \mathrm{~mol}^{-1}\right)$ [hydrogenated poly(butadiene)] which was supplied by TOTAL Cray Valley. Solvents were purchased from Fisher Scientific and used as received. Additionally, tetrahydrofuran (THF) was distilled from sodium and benzophenone prior to use, where anhydrous THF was needed. For cytotoxicity studies, polyurethane containing $0.1 \%(\mathrm{w} / \mathrm{w})$ zinc diethyldithiocarbamate (PU-ZDEC) and polyethylene (PE) were obtained from Hatano Research Institute, Food and Drug Safety Center, Japan. Fetal bovine serum and streptomycin were purchased from Gibco ${ }^{\mathrm{TM}}$.

\section{Instrumentation and Methods}

\section{Polymer Analysis}

${ }^{1} \mathrm{H}$ NMR spectroscopy (400 MHz) and ${ }^{13} \mathrm{C}$ NMR spectroscopy (100 MHz) were recorded with either a Bruker Nanobay 400, or Bruker DPX 400, using an appropriate deuterated solvent. Gel permeation chromatography (GPC) analysis was obtained by an Agilent Technologies 1260 Infinity systems in THF and polystyrene standards were used for calibration. Differential Scanning Calorimetry (DSC) thermograms were obtained using a TA DSC Q2000 adapted with a TA Refrigerated Cooling System 90, using aluminium TA Tzero pans and lids. Thermal transitions were recorded using heating and cooling rates of $10{ }^{\circ} \mathrm{C} \mathrm{min}-1$ and $20{ }^{\circ} \mathrm{C} \mathrm{min}^{-1}$, respectively. Tensile tests were carried out using AML instruments $^{\mathrm{TM}}$ single column tensiometer. From each film 3 strips $(5 \mathrm{~mm} \times 40 \mathrm{~mm} \times 1 \mathrm{~mm})$ were cut and individual samples were located between the grips of tensile instrument and elongated at a rate of $10 \mathrm{~mm} \min ^{-1}$ and the stress $v s$. strain curve recorded. The modulus of toughness was calculated by integrating the recorded plot to give the area under the curve. The trapezium rule was applied to calculate the area between zero strain to strain at break for each sample. The error reported is the standard deviation between the calculated areas of three repeats for each sample. Rheological experiments were carried out using a Malvern Panalytical Kinexus Lab+ fitted with a peltier plate cartridge and $8 \mathrm{~mm}$ parallel plate geometry. Cast samples were first heated to $60{ }^{\circ} \mathrm{C}$ applying $1 \mathrm{~N}$ of force to obtain good contact between the sample and geometry/plate. An amplitude sweep (between $0.1-16 \%$ at $1 \mathrm{~Hz})$ and frequency sweep $(0.01-100 \mathrm{~Hz})$ was then conducted to determine the linear viscoelastic regime as determined by the software (Malvern rSpace). The sample was then heated from $0{ }^{\circ} \mathrm{C}$ to $170{ }^{\circ} \mathrm{C}$ whilst recording the storage and loss moduli in oscillatory mode. To carry out rheology experiments on printed samples, they were loaded on the instrument at $25{ }^{\circ} \mathrm{C}$ and the amplitude and 
frequency sweep experiments were conducted at the same temperature. Variable temperature infrared spectra were recorded with Perkin-Elmer Spectrum 100 FTIR spectrometer equipped with Specac variable temperature cell holder and Temperature Controller. To carry out the experiment, $\mathrm{KBr}$ disks containing $1 \mathrm{wt} \%$ of each sample were prepared. Briefly, $3.00 \mathrm{mg}$ of each analyte was dissolved in minimum amount of THF and mixed with $300 \mathrm{mg} \mathrm{KBr}$ powder. The mixture was then dried completely under vacuum and grounded to achieve a fine powder which was then pressed to produce a disk. A pure $\mathrm{KBr}$ disk was also prepared following the same approach in order without adding a sample in order to collect the background. The carbonyl and NH regions of the collected spectra were fitted to Gaussian distributions. These were then used to calculate the area corresponds to each peak. The calculated areas are plotted in Figure S11.

\section{Biocompatibility Studies}

In vitro cell culture studies were performed using a mouse fibroblast cell line (L929, ECACC). Cells were cultured in Dulbecco's modified eagle's medium (high glucose) supplemented with $2 \mathrm{mM}$ of L-glutamine, $10 \%$ fetal bovine serum, penicillin $\left(100\right.$ units $\left.\mathrm{mL}^{-1}\right)$ and streptomycin $\left(0.1 \mathrm{mg} \mathrm{mL}^{-1}\right)$. Cells were maintained in a $5 \% \mathrm{CO}_{2}$ humidified atmosphere at $37^{\circ} \mathrm{C}$. Potential in vitro cytotoxicity effects of SPU1 were evaluated as follows. The polymeric films were incubated with tissue culture medium (3 $\mathrm{cm}^{2} \mathrm{~mL}^{-1}$, for 24 hours) as previously reported. ${ }^{32}$ Polyurethane containing $0.1 \%(\mathrm{w} / \mathrm{w})$ zinc diethyldithiocarbamate (PU-ZDEC) and polyethylene (PE) were used as positive and negative controls, respectively. After incubation, the polymeric films were removed and their liquid extracts (the culture media containing any materials leached from the polymeric films during the incubation) were either applied or diluted, where required, using the tissue culture medium prior to their application onto the cells. Cells were seeded in a 96 well plate at $1 \times 10^{4}$ cells per $\mathrm{mL}$ in tissue culture medium and allowed to adhere for 24 hours. The medium was then replaced with $100 \mu \mathrm{L}$ of either: (a) the tissue culture medium, (b) liquid extracts from SPU1 at different dilutions $(100,75,50,25 \%)$ (c) liquid extracts of the positive control (PU-ZDEC) at (100\%) or (d) liquid extracts of the negative control PE at (100\%), and cells incubated with the extracts for 48 hours. All diluted liquid extracts were sterile filtered prior to their application onto the cells. After 43h incubation, a 3-[4,5-dimethylthiazol-2-yl]-2,5diphenyltetrazolium bromide (MTT) solution $\left(5 \mathrm{mg} \mathrm{mL}^{-1}\right.$ in PBS) was added to the wells $(20 \mu \mathrm{L}$ per well) and cell viability assessed as previously reported. ${ }^{32}$

\section{Formulation Analysis}

Drug containing formulations for 3D printing were prepared in accordance with Table 1. Briefly, SPU1 and PEG $\left(20 \mathrm{~kg} \mathrm{~mol}^{-1}\right)$ were dispersed in ethyl acetate $\left(0.15 \mathrm{~mL} \mathrm{~g}^{-1}\right)$ and the formulation heated to $100{ }^{\circ} \mathrm{C}$ until homogenous. Paracetamol (acetaminophen) was then added to the translucent and yellow formulations and stirred under continued heating until evenly dispersed to yield pale yellow and opaque materials with $4 \mathrm{wt} \%$ PEG (F1) and 8 wt\% PEG (F2), respectively. The solvent was then removed in 
vacuo at $70{ }^{\circ} \mathrm{C}$ for 24 hours until constant mass was obtained. The formulations for 3D printing were loaded into an aluminium extrusion printing cartridge fitted with a sterile precision conical nozzle (I\&L Biosystems Ltd., Seer Green, UK) with an inner diameter of 0.609mm (21G). A Cellink INKREDIBLE printer was used to print the formulations. The extrusion pressure and temperature were set as 3.0 bar (300kPa), $100{ }^{\circ} \mathrm{C}$ and $3.1 \mathrm{bar}(310 \mathrm{kPa}), 105^{\circ} \mathrm{C}$ for formulation $\mathbf{F 1}$ and $\mathbf{F} 2$ respectively, a printing layer height of $0.3 \mathrm{~mm}$, printing speed of $10 \mathrm{~mm} \mathrm{~s}^{-1}$ and an infill of $100 \%$. A bar geometry $(30 \mathrm{~mm} \mathrm{x} 3 \mathrm{~mm} \mathrm{x}$ $2.5 \mathrm{~mm}$ ) was designed using Blender computer graphics software. G-codes were then generated with Slic3r (version 1.3.0) with the optimised printing parameters stated above.

Infrared spectra of pure paracetamol, SPU1, PEG $20 \mathrm{~kg} \mathrm{~mol}^{-1}$ and the two printed formulations (F1 and F2) were collected using a Perkin Elmer Frontier FTIR-ATR spectrometer between $4000 \mathrm{~cm}^{-1}$ and $550 \mathrm{~cm}^{-1}$ with a scan resolution of $2 \mu \mathrm{m}$ and a step size of $0.5 \mathrm{~cm}^{-1}$. The PXRD patterns of pure paracetamol, excipients (SPU1, and PEG) and the printed formulations (F1 and F2) were obtained at room temperature using a Bruker D8 Advance with DaVinci XRD instrument, setup in divergent beam mode running in Bragg-Brentano geometry and a Lynxeye 1D detector. A generator voltage of $40 \mathrm{kV}$ was set, with a current of $40 \mathrm{~mA}$. Samples were scanned over 2 theta range of $10^{\circ}$ to $40^{\circ}$ in a step size of $0.02^{\circ}$ and time per step of 1 second.

Small angle X-ray scattering (SAXS) data were collected using a Bruker Nanostar instrument with an Incoatec microfocus X-ray source operating at $45 \mathrm{kV}$. Scattering patterns were collected using a Vantec area detector $(2048 \times 2048$ pixels $)$ using silver behenate as a calibrant.

\section{Release Studies}

Dissolution testing of the printed implants was carried out using a Copely Scientific Dissolution Tester DIS 8000 with rotating USP I baskets according to USP specifications for paracetamol. Briefly, $0.05 \mathrm{M}$ potassium phosphate dissolution media was prepared with potassium dihydrogen phosphate in ultrapure water $(18.2 \mathrm{M} \Omega . \mathrm{cm})$ and adjusted to $\mathrm{pH} 6.8$ with a $50 \mathrm{wt} \%$ sodium hydroxide solution. Testing was carried out at constant volume at $37^{\square} \mathrm{C}$ in $900 \mathrm{~mL}$ of media. Five millilitre sample aliquots were removed at predetermined times $(24 \mathrm{~h}, 48 \mathrm{~h}, 72 \mathrm{~h}, 144 \mathrm{~h}, 168 \mathrm{~h})$ and replaced with five millilitres of media at $37^{\circ} \mathrm{C}$ and filtered with a $(0.45 \mu \mathrm{m}$ Millex PTFE hydrophilic filter). Samples were then analysed with an Agilent HPLC Series 1260 Infinity system, equipped with a UV diode array detector. A wavelength of $243 \mathrm{~nm}$ was used to quantify the paracetamol. The mobile phase compositions were $80 \%$ ultrapure water and 20\% HPLC grade methanol. An ACE C18-AR analytical column $(100 \mathrm{~mm} \times 4.6 \mathrm{~mm})$ with $5 \mu \mathrm{m}$ particle size was used to separate the samples at $30{ }^{\circ} \mathrm{C}$ using flowrate of $0.5 \mathrm{~mL} \mathrm{~min}^{-1}$. A $5 \mu \mathrm{L}$ injection volume was implemented for the samples; sample runtimes were 5 minutes and the paracetamol retention time was 1.62 minutes. Paracetamol stock solutions (160 ppm) were prepared by dissolving paracetamol (nominally $40 \mathrm{mg}$ ) in $5 \mathrm{~mL}$ methanol and diluting to volume with pH 6.8 dissolution media in a $250 \mathrm{~mL}$ volumetric flask. Standards were prepared with the $160 \mathrm{ppm}$ 
paracetamol stock solution and dissolution media. A typical standard calibration curve is included in the supplementary information (Figure S21).

Scanning Electron Microscope (SEM) imaging of the printed prototype implants were collected by cooling the printed bars to $-80{ }^{\circ} \mathrm{C}$ in a freezer and were cross-sectioned using a scalpel. The samples were then mounted on double-sided conductive carbon tape and sputter coated with gold with an Agar Sputter Coater. Imaging was carried out on A Hitachi SEM TM3030 Tabletop on a tilt stage. The microscope was equipped with a Bruker Scan generator, X Flash SDD detector, Bruker Nano MinSVE processor, Deben USB Chamberscope and Deben Sprite HR Stage controller joystick.

\section{Acknowledgements}

The authors would like to acknowledge funding from the EPSRC (EP/N024818/1) in support of a postdoctoral fellowships for LRH, EAC, SK, YW. Additionally, we acknowledge the University of Reading (ref GS17-025) for postgraduate studentship in support of SS and the EPSRC Centre for Doctoral Training in Additive Manufacturing and 3D Printing (EP/L01534X/1) in support of MIEB. The authors would also like to thank the University of Reading for access to analytical instrumentation within the Chemical Analysis Facility, Mr Nicolas Michael for his expertise in mass spectrometry, Professor Kenneth Shankland for advice on PXRD, and Dr Barnaby Greenland for useful discussions relating to polyurethanes. We acknowledge TOTAL Cray Valley for the supply of Krasol ${ }^{\mathrm{TM}}$ HLBHP2000.

\section{References}

1 A. S. Narang and S. H. Boddu, in Excipient Applications in Formulation Design and Drug Delivery, eds. A. S. Narang and S. H. Boddu, Springer, 2015, pp. 1-10.

2 S. P. Chaudhari and P. S. Patil, Int. J. Adv. Pharmacy, Biol. Chem., 2016, 1, 21-34.

3 S. Pundir, A. Badola and D. Sharma, Int. J. Drug Res. Technol., 2013, 3, 12-20.

4 M. vallet-Regi, F. Balas and D. Arcos, Angew. Chemie - Int. Ed., 2007, 46, 7548-7558.

5 Y. Cui, B. Li, H. He, W. Zhou, B. Chen and G. Qian, Acc. Chem. Res., 2016, 49, 483-493.

6 J. Della Rocca, D. Liu and W. Lin, Acc. Chem. Res., 2011, 44, 957-968.

$7 \quad$ R. Duncan, Nat. Rev. Drug Discov., 2003, 2, 347-360.

8 M. A. C. Stuart, W. T. S. Huck, J. Genzer, M. Müller, C. Ober, M. Stamm, G. B. Sukhorukov, I. Szleifer, V. V. Tsukruk, M. Urban, F. Winnik, S. Zauscher, I. Luzinov and S. Minko, Nat. Mater., 2010, 9, 101-113.

9 W. B. Liechty, D. R. Kryscio, B. V. Slaughter and N. A. Peppas, Annu. Rev. Chem. Biomol. 
Eng., 2010, 1, 149-173.

10 K. Y. Lee and D. J. Mooney, Chem. Rev., 2001, 101, 1869-1879.

11 P. Gupta, K. Vermani and S. Garg, Drug Discov. Today, 2002, 7, 569-579.

12 O. S. Fenton, K. N. Olafson, P. S. Pillai, M. J. Mitchell and R. Langer, Adv. Mater., 2018, 30, 1705328.

13 O. Pillai and R. Panchangnula, Curr. Opin. Chem. Biol., 2001, 5, 447-451.

14 K. E. Uhrich, S. M. Cannizzaro, R. S. Langer and K. M. Shakesheff, Chem. Rev., 1999, 99, 3181-3198.

15 W. N. Sivak, J. Zhang, S. Petoud and E. J. Beckman, Acta Biomater., 2009, 5, 2398-2408.

16 G. V. Salmoria, P. Klauss, K. M. Zepon and L. A. Kanis, Int. J. Adv. Manuf. Technol., 2013, 66, 1113-1118.

17 J. Intra, J. M. Glasgow, H. Q. Mai and A. K. Salem, J. Control. Release, 2008, 127, 280-287.

18 R. Chen, G. Li, A. Han, H. Wu and S. Guo, J. Biomater. Sci. Polym. Ed., 2016, 27, 529-543.

19 D. Q. M. Craig, Drug Dev. Ind. Pharm., 1990, 16, 2501-2526.

20 G. Fontana, M. Licciardi, S. Mansueto, D. Schillaci and G. Giammona, Biomaterials, 2001, 22, 2857-2865.

21 A. L. Acton, C. Fante, B. Flatley, S. Burattini, I. W. Hamley, Z. Wang, F. Greco and W. Hayes, Biomacromolecules, 2013, 14, 564-574.

22 W. J. Jia, Y. C. Gu, M. Gou, M. Dai, X. Li, B. Kan, J. L. Yang, Q. F. Song, Y. Q. Wei and Z. Y. Qian, Drug Deliv., 2008, 15, 409-416.

23 T. Patrício, M. Domingos, A. Gloria, U. D’Amora, J. F. Coelho and P. J. Bártolo, Rapid Prototyp. J., 2014, 20, 145-156.

24 Y. Zhang and R. X. Zhuo, Biomaterials, 2005, 26, 6736-6742.

25 G.-H. Son, B.-J. Lee and C.-W. Cho, J. Pharm. Investig., 2017, 47, 287-296.

26 Y. Gu, X. Chen, J. H. Lee, D. A. Monteiro, H. Wang and W. Y. Lee, Acta Biomater., 2012, 8, 424-431.

27 J. Yu, Y. Xu, S. Li, G. V. Seifert and M. L. Becker, Biomacromolecules, 2017, 18, 4171-4183.

28 H. Patil, R. V. Tiwari and M. A. Repka, AAPS PharmSciTech, 2016, 17, $20-42$.

29 A. Goyanes, F. Fina, A. Martorana, D. Sedough, S. Gaisford and A. W. Basit, Int. J. Pharm., 2017, 527, 21-30.

30 S. A. Khaled, M. R. Alexander, R. D. Wildman, M. J. Wallace, S. Sharpe, J. Yoo and C. J. Roberts, Int. J. Pharm., 2018, 538, 223-230.

31 A. Feula, A. Pethybridge, I. Giannakopoulos, X. Tang, A. Chippindale, C. R. Siviour, C. P. Buckley, I. W. Hamley and W. Hayes, Macromolecules, 2015, 48, 6132-6141.

32 A. Feula, X. Tang, I. Giannakopoulos, A. M. Chippindale, I. W. Hamley, F. Greco, C. P. Buckley, C. R. Siviour, W. Hayes, C. Paul Buckley, C. R. Siviour and W. Hayes, Chem. Sci., 2016, 7, 4291-4300. 
D. Hermida-Merino, A. Feula, K. Melia, A. T. Slark, I. Giannakopoulos, C. R. Siviour, C. P. Buckley, B. W. Greenland, D. Liu, Y. Gan, P. J. Harris, A. M. Chippindale, I. W. Hamley and W. Hayes, Polymer (Guildf)., 2016, 107, 368-378.

S. Salimi, L. R. Hart, A. Feula, A. B. R. Touré, E. A. Kabova, L. Ruiz-Cantu, D. J. Irvine, R. Wildman, K. Shankland and W. Hayes, Eur. Polym. J., 2019, 118, 88-96.

R. J. Wojtecki, M. A. Meador and S. J. Rowan, Nat. Mater., 2011, 10, 14-27.

J. S. Maximilien, in Handbook of Pharmaceutical Excipients, eds. R. C. Rowe, P. J. Sheskey and M. E. Quinn, Pharmaceutical Press, 6th edn., 2009, pp. 522-524.

A. A. D'souza and R. Shegokar, Expert Opin. Drug Deliv., 2016, 13, 1257-1275.

P. Woodward, D. H. Merino, B. W. Greenland, I. W. Hamley, Z. Light, A. T. Slark, W. Hayes and D. Hermida-Merino, Macromolecules, 2010, 43, 2512-2517.

P. Woodward, A. Clarke, B. W. Greenland, D. Hermida-Merino, L. Yates, A. T. Slark, J. F. Miravet, W. Hayes and D. H. Merino, Soft Matter, 2009, 5, 2000-2010.

R. P. Sijbesma, F. H. Beijer, L. Brunsveld, B. J. B. Folmer, J. H. K. Ky. Hirschberg, R. F. M. M. Lange, J. K. L. Lowe, E. W. Meijer, Science, 1997, 278, 1601-1604.

41 P. Cordier, F. Tournilhac, C. Soulié-Ziakovic and L. Leibler, Nature, 2008, 451, 977-980.

42 V. Simic, L. Bouteiller and M. Jalabert, J. Am. Chem. Soc., 2003, 125, 13148-13154.

43 S. Sivakova, D. A. Bohnsack, M. E. Mackay, P. Suwanmala and S. J. Rowan, J. Am. Chem. Soc., 2005, 127, 18202-18211.

44 K. Hackethal, F. Herbst and W. H. Binder, J. Polym. Sci. Part A Polym. Chem., 2012, 50, 4494-4506.

45 D. J. Skrovanek, S. E. Howe, P. C. Painter and M. M. Coleman, Macromolecules, 1985, 18, 1676-1683.

46 D. Hermida-Merino, A. T. Slark, H. M. Colquhoun, W. Hayes and I. W. Hamley, Polym. Chem., 2010, 1, 1263-1271.

47 J. Yang, H. Chen, Y. Yuan, D. Sarkar and J. Zheng, Front. Chem. Sci. Eng., 2014, 8, 498-510.

48 L. R. Hart, S. Li, C. Sturgess, R. Wildman, J. R. Jones and W. Hayes, ACS Appl. Mater. Interfaces, 2016, 8, 3115-3122.

49 S. A. Khaled, J. C. Burley, M. R. Alexander, J. Yang and C. J. Roberts, Int. J. Pharm., 2015, 494, 643-650.

50 S. A. Khaled, M. R. Alexander, D. J. Irvine, R. D. Wildman, M. J. Wallace, S. Sharpe, J. Yoo and C. J. Roberts, AAPS PharmSciTech, 2018, 19, 3403-3413.

51 S. A. Khaled, M. R. Alexander, R. D. Wildman, M. J. Wallace, S. Sharpe, J. Yoo and C. J. Roberts, Int. J. Pharm., 2018, 538, 223-230. USP 40 - NF 35 The United States Pharmacopeia and National Formulary 2017, United States Pharmacopeial Convention, 2016.

53 I. S. Khattab, A. Nada and A. A. Zaghloul, Drug Dev. Ind. Pharm., 2010, 36, 893-902. 
54 H. A. Moynihan and I. P. O’Hare, Int. J. Pharm., 2002, 247, 179-185.

55 M. Haisa, S. Kashino, R. Kawai and H. Maeda, Acta Crystallogr. Sect. B Struct. Crystallogr. Cryst. Chem., 1976, 32, 1283-1285.

56 I. Yilgor and E. Yilgor, Polym. Rev., 2007, 47, 487-510.

57 S. Burattini, H. M. Colquhoun, B. W. Greenland and W. Hayes, Faraday Discuss., 2009, 143, 251-264. 